\title{
Particle Filtering in the Design of an Accurate Pupil Tracking System
}

\author{
Morteza Sadri \\ Islamic Azad University Central \\ Tehran Branch \\ IAUCTB, Orak St. Hamila Blvd. \\ Poonak Sq. Tehran, Iran
}

\author{
Kaveh Kangarloo \\ Islamic Azad University Central \\ Tehran Branch \\ IAUCTB, Orak St. Hamila Blvd. \\ Poonak Sq. Tehran, Iran
}

\author{
Fardad Farokhi \\ Islamic Azad University Central \\ Tehran Branch \\ IAUCTB, Orak St. Hamila Blvd. \\ Poonak Sq. Tehran, Iran
}

\begin{abstract}
Accurate pupil tracking plays an important role in many sophisticated applications such as $\mathrm{HCI}^{1}$, behavioral research, driver's fatigue detection and marketing. In this study particle filter helps us to track pupil in video frames under active IR illumination. The proposed method can be categorized as follow; The eye video is captured by a $\mathrm{HMD}^{2}$ camera, then precise location of the pupil is determined by thresholding and applying Snake algorithm, next the contour of the detected pupil is modeled with an ellipse, finally the parameters of the ellipse such as center coordinates and major \& minor axiss are tracked by particle filter. Experimental results of the proposed method which applied to 20 different subjects, demonstrate the accuracy of the proposed method.
\end{abstract}

\section{General Terms}

Human-Computer Interaction

\section{Keywords}

Pupil tracking, Snake algorithm, HMD camera, Particle filter

\section{INTRODUCTION}

Tracking pupil is one of the prevailing applications of image processing. Pupil tracking refers to precise locating and tracking of pupil and its center by features like, shape, color, size and distance between two pupils. Pupil tracking has widespread applications in medical, biometrics, behavioral research, $\mathrm{HCI}^{2}$ and so on.

During reading or watching a scene, eye movements reveal useful information about how everyone processes the received visual data to confirm it with his knowledge. Eye tracking can be used for psychologic studies, human and machine interface, driver fatigue detection and so on.

The term eye tracking used in this research refers to gaze determination or tracking eye movement relative to the head. Collected information like eye position and movement give us movement pattern of the eye.

There is a variety of pupil tracking algorithms with different accuracies and applications. But we can categorize them in two main groups; intrusive and non-intrusive methods. intrusive methods track eye by contacting some sensors or electrodes to the eye or skin around eye. Though these method offer high accuracy in tracking, but they are not user friendly and hard to setup.

${ }^{1}$ Human Computer Interaction

${ }^{2}$ Head Mounted Display
In the other hand, non-intrusive methods, are more user friendly, less expensive, more flexible and easily performable. Since non-intrusive methods are video-based, main part of them is a video camera connected to a PC for real-time image processing.

\section{RELATED WORKS}

Numerous researches have been conducted in the field of eye tracking and yield to different methods and applications that will be mentioned below.

In 2011 Liaghatdar et al. (12) made an eye tracking system for investigating person behavioral changes in video. User's eye video captured by a camera mounted in front of the eye. Pupil is detected and segmented by Otsu method and genetic algorithm respectively. Circular Hough transform was used to modeling pupil and kalman filter was exploited for tracking and compensating generated errors in previous steps. Investigating results by a professional can leads to detection of person reaction by pupil movements.

In 2008 Panev et al. (13) tracked eye by modeling pupil with a standard ellipse then approximate position of pupil detected by $\mathrm{GLD}^{3}$ and a particle filter with limited particles used to track pupil.

In 2002 Zhu and Ji. (16) made a real time eye tracking by combining kalman filter and mean shift under IR illumination. Pupil is illuminated by IR across camera optical axis, so the bright pupil is created and pupil is seen as a bright spot in the image. Then pupil is tracked by kalman filter and if fails, mean shift will complete tracking process.

In 2011 Jian-nan et al. (9) tracked pupil by particle filter for gaze tracking. A fixed camera with IR illumination used to create bright pupil due to approximate pupil detection. Then a morphology operator is applied to determine accurate contour of pupil and pupil is modeled by an ellipse. Finally, particle filter will track ellipse parameters.

In $2002 \mathrm{Ji}$ et al. (8) tracked eye, gaze and face pose to monitor driver's vigilance. They used $\mathrm{NIR}^{4}$ illumination to creating bright pupil in order to approximate pupil location. By adjusting IR illuminator, bright and dark pupil is obtained and by image subtraction, location of pupil is approximated and thresholding by Kullback information distance results in exact detection of pupil, finally kalman filter is exploited for tracking.

\footnotetext{
${ }^{3}$ Gray Level Differences
}

${ }^{4}$ Near Infrared 
In 2007 Komogortsev et al. (11) developed an eye controlled computer interface with kalman filter. In this research an attention focus kalman filter (AFKF) that makes an eye movement language to real-time perception and modeling of human visual system. In this method, basic movements of eye are determined and point of attraction of person is predicted.

In 2010 Asadifard andShabanzadeh (2) made an adaptive detection of pupil center by face detection and analyzing accumulative distribution function. In this method face location is approximated by viola-jones face detector. Eye is located by its structural information. By calculating histogram of the region and using accumulative distribution function, eyelashes and eyelids removed in an adaptive manner. Finally the center of this region is supposed as the pupil center.

\section{HUMAN'S EYE ANATOMY AND EYE MOVEMENT}

The human eye is like a camera. Light comes in through the cornea, a clear cover that is like the glass of a camera's aperture. The amount of light coming in is controlled by the pupil, an opening that opens and closes a little like a camera shutter. The light focuses on the retina, a series of lightsensitive cells lining the back of the eye. The retina acts like camera film, reacting to the incoming light and sending a record of it via the optic nerve to the brain.

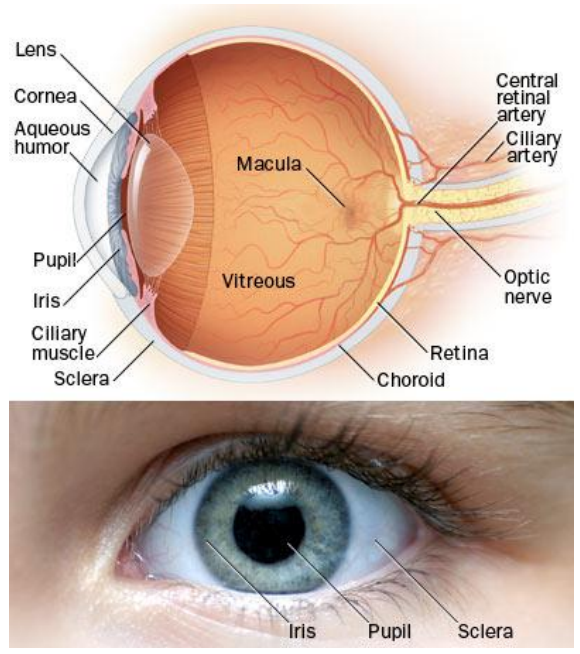

Fig. 1: human eye

Eye movement is the voluntary or involuntary movement of the eyes, helping in acquiring, fixating and tracking visual stimuli. It may also compensate for a body movement, such as when moving the head.

There are three main basic types of eye movements:

Vergence Movements or convergence are the movements of both eyes to make sure that the image of the object being looked at falls on the corresponding spot on both retinas.

Saccades are the rapid movement of eyes that is used while scanning a visual scene. During each saccade the eyes move as fast as they can and the speed cannot be consciously controlled in between the stops. ${ }^{8]}$. The movements are worth a few minutes of arc, moving at regular intervals about three to four per second. Pursuit Movements or Smooth pursuit are the movements that the eyes make while tracking an object's movement, so that its moving image can remain maintained on fovea

\section{METHODS}

The aim of this study is to find a precise pupil tracking algorithm by active contours and particle filter in order to use obtained information sophisticated applications such as HCI, behavioral research, medicals and so on.

Pupil tracking algorithm used in this research consists of four stages that can be seen in figure 3 respectively. As we know quality of eye tracking performance, directly related to the quality of captured images from camera, for this reason we used infrared illuminator for imaging. Using infrared for imaging, not only improve imaging quality but also minimize ambient light sources effects. In addition whereas infrared is not visible for human, making least interference with other activities. But this is not the whole story, because in spite of infrared benefits, our image will suffer from corneal reflections which may degrade pupil detection accuracy and cause faults in detection step. Hence by applying a circular average filter and choosing appropriate search radius, algorithm can omit disturbing reflections.

Another auxiliary tool for increasing the quality of imaging at the same time reducing computational complexity is HMD camera. As you see in figure 2 camera is mounted on a helmet and the camera situated in front of one eye. By focusing camera on the eye, we have only the eye image, so the captured image has better resolution and there is no need for the detection algorithm to search for the eye in the face image.

The next stage and one of the most important one, is pupil detection, because without having correct information about pupil's position, next operations can't be done and even a tiny error in this step, will ruin tracking procedure.

After pupil detection, we must model it. Pupil has two distinct features; dark color and circular form that ease modeling of pupil. Modeling pupil helps us to reduce processing load of the system by tracking only the model parameter instead of tracking every point of pupil.

Finally we must track model parameters frame by frame in order to locate pupil in each frame precisely and monitor pupil changes and its moving pattern.
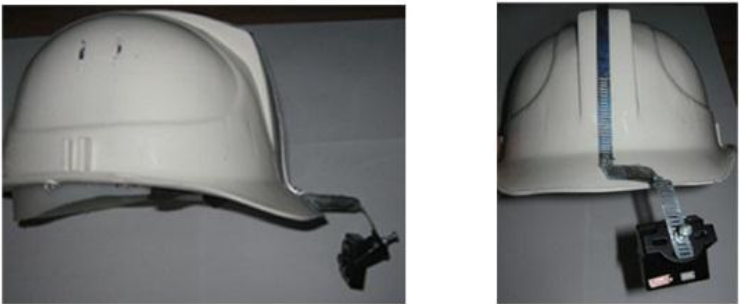

Fig. 2: head fixture

\subsection{Eye detection and modeling}

In order to detect pupil, we first make a rough estimate of pupil using variable thresholding. We start thresholding by low threshold level and in each step, shape feature and circularity of pupil are determined, thresholding continues till circularity condition is met. After estimating pupil position we should define exact boundaries of pupil. In our proposed algorithm active contours or snakes is applied to detect the accurate pupil circle, which consequently increases the performance of the tracking algorithm in terms of accuracy 


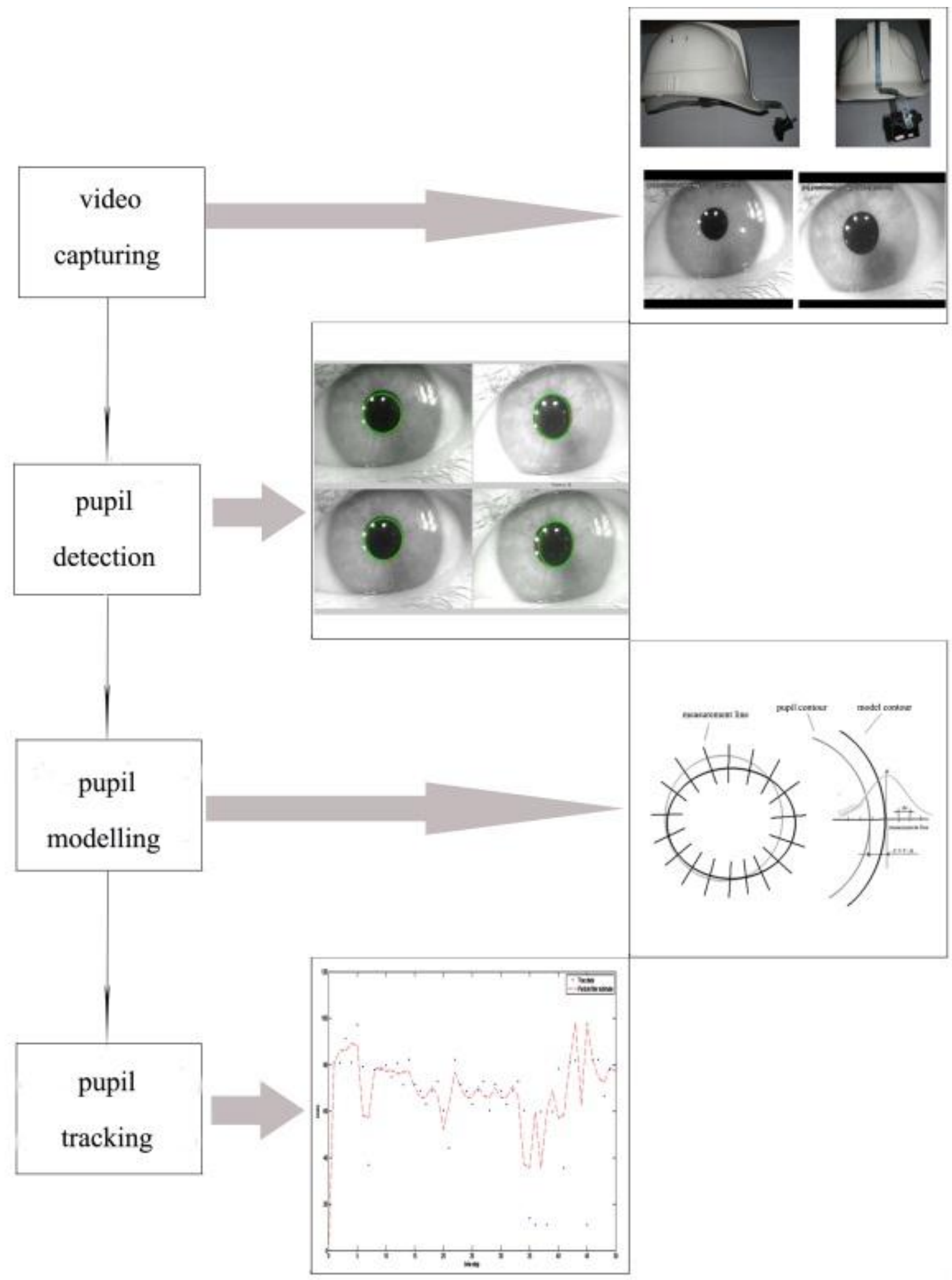

Fig. 3: pupil tracking block diagram 
and processing time. For detection of the exact contour of pupil, we will apply $\mathrm{n}$ snake points to initial contour of pupil, obtained by thresholding. The snake curve is deformed iteratively by minimizing the energy function, in each iteration a new snake curve with lower energy and better fitting to the pupil boundary is obtained. Having precise contour of pupil we are ready to model the pupil and it helps us reducing computational complexity of the algorithm, because by tracking the model parameters we can reproduce pupil. Unlike what is assumed, pupil always is not like a complete circle and mostly near to an ellipse. If we model pupil to a circle, we might lose some marginal information of pupil and tracking accuracy will be degraded. Consequently we used an ellipse with five dimensional vector. Ellispse parameters are as follow:

$\vec{x}=\left[c_{x}, c_{y}, \lambda_{1}, \lambda_{2}, \theta\right]$, where $\left(c_{x}, c_{y}\right)$ is the center of the pupil, $\lambda_{1}, \lambda_{2}$ are the major and minor axes, and $\theta$ is the angle of the major axis with respect to the vertical coordinate. This vector represents the state of the system.

\subsection{Eye tracking}

The final and the most important stage of this algorithm, is tracking of pupil with particle filter. Particle filters approximate the continuous conditional $\operatorname{pdf} p\left(\mathbf{x}_{k} \mid \mathbf{Z}_{k}\right)$ by an independent set of random samples (also known as particles) $\left\{x_{k}^{i}\right\}_{i=1}^{N}$ and associated positive weights $\left\{w_{k}^{i}>\right.$ $0\}_{i=1}^{N}$.

The PF is an algorithm for propagating and updating the samples and weights numerically such that the random samples are approximately distributed as independent samples arising from the $\operatorname{pdf} p\left(\mathbf{x}_{k} \mid \mathbf{Z}_{k}\right)$. The numerical approximation for the prediction step is

$p\left(\begin{array}{ll}\mathbf{x} & k\end{array} \mid \begin{array}{ll}\mathbf{Z} & k-1\end{array}\right)$

$\int p\left(\begin{array}{ll}\mathbf{x}_{k} & \mathbf{x}_{k-1}\end{array}\right) p\left(\begin{array}{ll}\mathbf{x}_{k-1} \mid \mathbf{Z}_{k-1} & \end{array}\right) d x_{k-1}$

$\approx(1 / \mathrm{N}) \sum_{i=1}^{N} p\left(\begin{array}{ll}\mathbf{x}_{k} \mid \mathbf{x} \quad{ }_{k-1}=\mathbf{x}_{k-1}^{i} & \end{array}\right)$

Where $\left\{x_{k-1}^{i}\right\}_{i=1}^{N} \quad$ are $N$ independent, identically distributed (iid) samples drawn from $p\left(\begin{array}{lll}\mathbf{x}_{k-1} \mid \mathbf{Z}{ }_{k-1}\end{array}\right)$.

The detailed steps of the PF with sampling importance resampling (SIR) are:

1. Select the number of particles $(N)$ to be generated and the threshold for resampling $\left(N_{\text {thres }}\right)$

\section{Initialization}

Set $k=1$.

Generate samples $\left\{x_{1}^{i}\right\}_{i=1}^{N}$ from the prior density $p_{0}\left(\mathrm{x}_{1}\right)$.

Set $\left\{w_{1}^{i}=1 / N\right\}_{i=1}^{N}$

3. Increment $k: k=k+1$

4. Prediction

Generate $N$ samples $\left\{w^{i}(k, k-1) \sim N(0, Q(K, K-1))\right\}_{i=1}^{N}$

Compute $N$ predicted state vectors: $\left\{w_{k}^{i}=\Phi(k, k-\right.$ 1) $\left.x_{k-1}^{i}+w^{i}(K, K-1)\right\}_{i=1}^{N}$

5. State update with measurement $\mathbf{z}_{k}$
Compute the likelihoods $:\left\{p\left(\mathbf{z}{ }_{k} \mid \mathbf{x}_{\boldsymbol{k}}^{\boldsymbol{i}}\right)\right\}_{i=1}^{N}$

Update the weights: $\left\{w_{k}^{i}=\frac{w_{k-1}^{i} p\left(\mathbf{z}{ }_{k} \mid \mathbf{x}_{k}^{i}\right)}{\sum_{i=1}^{N} w_{k-1}^{i} p\left(\mathbf{z} \quad k \mid \mathbf{x}_{k}^{i}\right)}\right\}{ }_{i=1}^{N}$

Compute the measurement updated state estimate: $x_{k \mid k}=$ $\sum_{i=1}^{N} w_{k}^{i} x_{k}^{i}$

6. Compute effective sample size $\left(N_{\text {eff }}\right)$

$N_{\text {eff }}=1 / \sum_{i=1}^{N}\left(w_{k}^{i}\right)^{2}$

If $N_{\text {eff }}>N_{\text {thres }}$, then

Go to step 3 .

Else

Resample a new set $\left\{\mathbf{x}_{\boldsymbol{k}}^{i}\right\}_{i=1}^{N}$ by sampling

with

replacement $N$ times from the discrete set

$\left\{\mathbf{x}_{\boldsymbol{k}}^{i}\right\}_{i=1}^{N}$ where $\operatorname{Pr}\left(\mathbf{x}_{\boldsymbol{k}}^{i}=\mathbf{x}_{\boldsymbol{k}}^{\boldsymbol{j}}\right)=\mathbf{w}_{\boldsymbol{k}}^{\boldsymbol{j}}$

Set $\left\{\mathbf{w}_{\boldsymbol{k}}^{j}=1 / N\right\}_{i=1}^{N}$

Go to step 3 .

End

5. EXPERIMENT SETUP AND RESULTS

Performance of the proposed algorithm in detection was examined on the CASIA-Iris V3.05 which over 700 of eye images went through pupil detection algorithm and in tracking, was examined on videos recorded by HMD camera from 20 persons with 25 fps and results are summarized in Table 1. All experiments were done by MATLAB R2008-a on a $2.8 \mathrm{GHz}$ PC with $4 \mathrm{~GB}$ of memory.

\section{DISCUSSION}

The main objective of this research was set to develop a low cost and simple eye tracking system based on pupil detection and continuous tracking of pupil with HMD camera that makes user move head freely in order to investigate pupil changes and studying pupil moving pattern. Precise detection of pupil by snakes and tracking by PF were key factors to our success.

One challenge using particle filter, is computational load of the filter that increases processing time and problem arises while using in real-time applications. One interesting offer for future development, is combining PF with IMM, so we can use different types of filter for different moving pattern of pupil in order to reduce computational cost.

${ }^{5}$ CASIA-IrisV3 contains a total of 22,035 iris images from more than 700 subjects. All iris images are 8 bit gray-level JPEG files, collected under near infrared illumination with a resolution of $320 * 280$ and $640 * 480$. 
Table 1. Numerical results of the pupil tracking algorithm

\begin{tabular}{|c|c|c|c|c|c|c|c|}
\hline Video Sampling rate & 24 & 24 & 24 & 24 & 24 & 24 & 24 \\
\hline Thresholding range & $0.1-1.0$ & $0.1-1.0$ & $0.1-1.0$ & $0.1-1.0$ & $0.1-1.0$ & $0.1-1.0$ & $0.1-1.0$ \\
\hline Snake points & 32 & 32 & 32 & 32 & 32 & 32 & 32 \\
\hline Snake iteration & 220 & 220 & 220 & 220 & 220 & 220 & 220 \\
\hline Number of particles & 10 & 30 & 60 & 100 & 200 & 300 & 500 \\
\hline Points to be tracked & 1000 & 1000 & 1000 & 1000 & 1000 & 1000 & 1000 \\
\hline Tracked points & 100 & 150 & 220 & 400 & 830 & 950 & 970 \\
\hline Processing time & 120 & 150 & 200 & 350 & 700 & 780 & 790 \\
\hline Accuracy & $12 \%$ & $15 \%$ & $22 \%$ & $40 \%$ & $83 \%$ & $95 \%$ & $97 \%$ \\
\hline
\end{tabular}
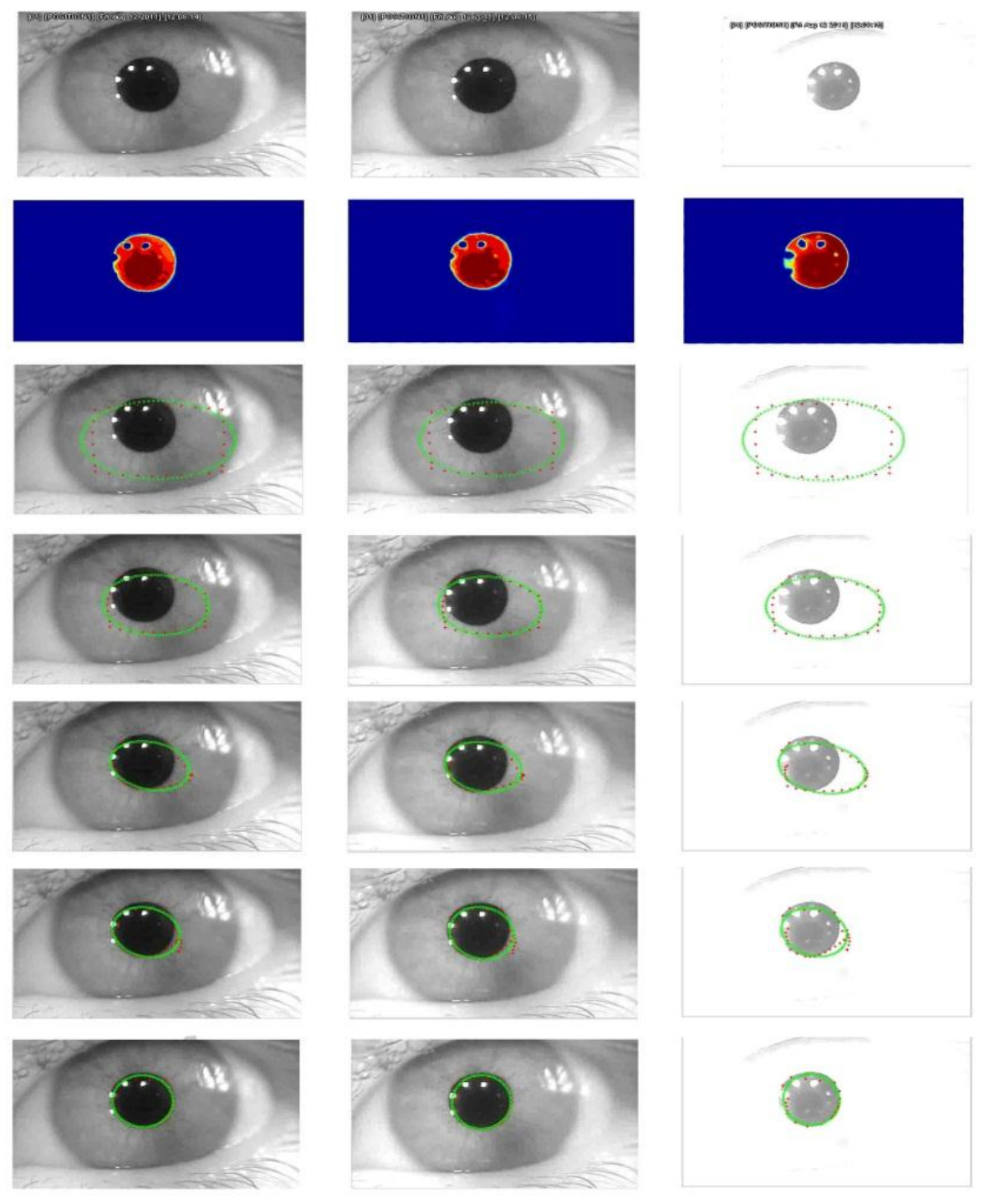

Fig. 4: Results of pupil detection stage 

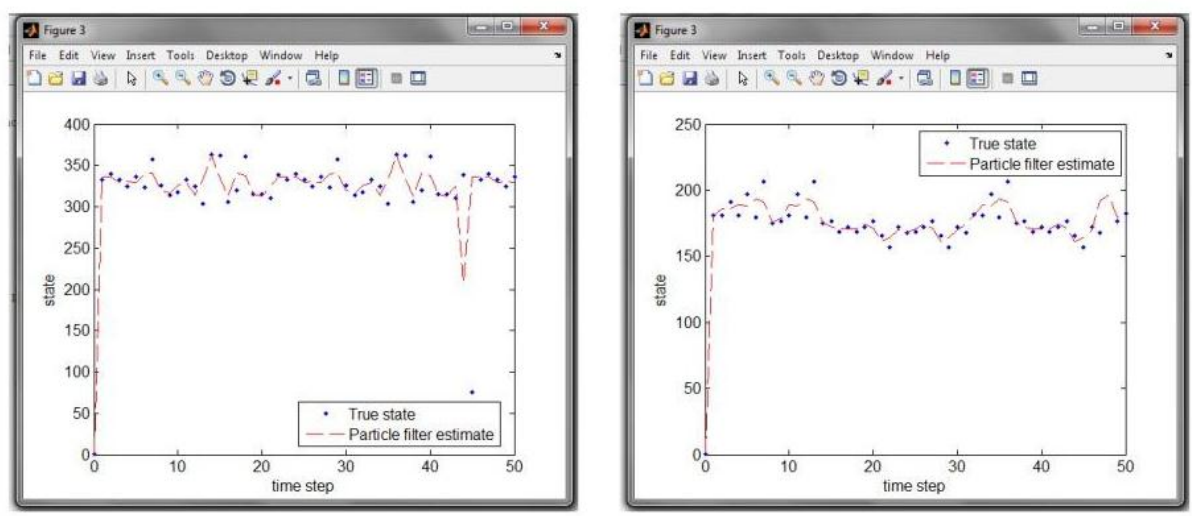

Fig. 5: Results of pupil tracking stage

\section{REFERENCES}

[1] M.S. Arulampalam, S. Maskell, N. Gordon, T. Clapp , 2002 "A Tutorial on Particle Filters for Online Nonlinear/Non-Gaussian Bayesian Tracking" IEEE Transactions On Signal Processing, Vol. 50, No. 2, February

[2] M. Asadifard, J. Shabanzadeh, 2010 " Automatic Adaptive Center of Pupil Detection Using Face Detection and CDF Analysis " Proceedings of the International MultiConference of Engineers and Computer Scientists, Vol I.

[3] CASIA V3.0 Iris Image Database http://www.cbsr.ia.ac.cn/IrisDatabase.htm

[4] H. Fang, J.W. Kim, J.W. Jang, 2011 "A Fast Snake Algorithm for Tracking Multiple Objects" Journal of Information Processing Systems, Vol.7, No.3, pp:519-530

[5] N.J. Gordon, D.J. Salmond, A.F.M. Smith, 1993 "Novel Approach to Nonlinear/Non-Gaussian Bayesian State Estimation" IEE Proceedings-F, Vol.140, No. 2 pp:107-113

[6] H. Hua, P. Krishnaswamy, 2006 "Video-based Eyetracking Methods and Algorithms in Headmounted Displays" OPTIC EXPRESS 4328, Vol. 14, No.10

[7] A.A. Jarjes . K. Wang, G.J.Mohammad, 2010 "GVF Snake-based Method for Accurate Pupil Contour Detection" Information Technology Journal 9(8):1653-1658, ISSNC1812-5638

[8] Q. Ji, X. Yang, 2002 "Real-Time Eye, Gaze, and Face Pose Tracking for Monitoring Driver Vigilance" Real-Time Imaging 8, 357-377
[9] C. Jian-nan, Z. Chuang, Q. Yan-jun, L. Ying, Y. Li, 2011 "Pupil Tracking Method Based on Particle Filtering in Gaze Tracking System" International Journal of the Physical Sciences Vol. 6(5), pp. $1233-1243$

[10] [M. Kass, A. Witkin, and D. Terzopoulos, 1987 "Snakes: Active Contour Models," Int. J. Comput. Vis., vol. 1, no. 4, pp. 321-331,

[11] O. V. Komogortsev, J. I. Khan, 2007 "Kalman Filtering in the Design of Eye-Gaze-Guided Computer Interfaces"J. Jacko (Ed.): HumanComputer Interaction, Part III, HCII 2007, NCS 4552, pp. 679-689, 2007. Springer-Verlag Berlin Heidelberg

[12] A. Liaghatdar, K. Kangarloo, F. Farokhi, 2011 "Pupil Localizing in Video images the First Step Toward Eye Monitoring" International Conference on Multimedia Technology (ICMT) pp: 3163-3166.

[13] S. Panev, O. Bombarov, S. Sokolov, 2008 " IR Based Pupil Tracking Using Particle Filtering" International Scientific Conference Computer Science'2008

[14] D. A. Robinson, 1963 "A method of measuring eye movements using a scleral search coil in a magnetic field,'IEEE Trans. Biomed. Electron. BME 10, 137145 .

[15] Y. Yu, Q. Cheng, 2006 "Particle Filters for Maneuvering Target Tracking Problem" Signal Processing, Vol.86, Issue1, pp: 195-203

[16] Z. Zhu, Q. Ji, K. Fujimura, 2002 "Combining Kalman Filtering and Mean Shift for Real Time Eye Tracking Under Active IR Illumination" IEEE , 1051-4651/02 